\title{
Geographical origin of Plasmodium vivax in the Republic of Korea: haplotype network analysis based on the parasite's mitochondrial genome
}

\author{
Moritoshi Iwagami ${ }^{+1}$, Seung-Young Hwang ${ }^{+2}$, Megumi Fukumoto 1,3, Toshiyuki Hayakawa4, Kazuyuki Tanabe4, So- \\ Hee Kim ${ }^{5}$, Weon-Gyu Kho*2,5 and Shigeyuki Kano*1,3
}

\begin{abstract}
Background: The Republic of Korea (South Korea) is one of the countries where vivax malaria had been successfully eradicated by the late 1970s. However, re-emergence of vivax malaria in South Korea was reported in 1993. Several epidemiological studies and some genetic studies using antigenic molecules of Plasmodium vivax in the country have been reported, but the evolutionary history of P. vivax has not been fully understood. In this study, the origin of the South Korean $P$. vivax population was estimated by molecular phylogeographic analysis.

Methods: A haplotype network analysis based on P. vivax mitochondrial (mt) DNA sequences was conducted on 11 . vivax isolates from South Korea and another 282 P. vivax isolates collected worldwide.

Results: The network analysis of P. vivax mtDNA sequences showed that the coexistence of two different groups (A and B) in South Korea. Groups A and B were identical or close to two different populations in southern China.

Conclusions: Although the direct introduction of the two P. vivax populations in South Korea were thought to have been from North Korea, the results of this analysis suggest the genealogical origin to be the two different populations in southern China.
\end{abstract}

\section{Background}

Malaria is distributed not only in tropical and subtropical areas but also in some temperate areas of the world. Plasmodium falciparum, which is distributed in tropical and subtropical areas, accounts for $90 \%$ of malaria cases. Like P. falciparum, Plasmodium vivax is distributed in tropical and subtropical areas, but its range extends to some temperate areas. In Asian and South American countries, the proportion of $P$. falciparum cases is gradually decreasing due to global malaria controls programmes, such as The Roll Back Malaria Partnership and The Global Fund. On the other hand, the proportion of P. vivax cases is gradu-

\footnotetext{
*Correspondence: wgkho@inje.ac.kr, kano@ri.imcj.go.jp

2 Department of Parasitology, Inje University, College of Medicine, 633-165 Gaegum-dong, Busanjin-gu, Busan 614-735, Korea

2 Department of Tropical Medicine and Malaria, Research Institute, National Center for Global Health and Medicine, 1-21-1 Toyama, Shinjuku, Tokyo 1628665, Japan

+ Contributed equally

Full list of author information is available at the end of the article
}

ally increasing [1]. Therefore, $P$. vivax should be given greater attention than it has received.

The Republic of Korea (South Korea) is one of the countries where vivax malaria had been successfully eradicated by the late 1970s. This was due to an effective national eradication programme conducted by the National Malaria Eradication Service under the operation of the South Korean government with the support of the WHO [2-4]. However, in 1993, the first case of indigenous vivax malaria after the eradication program was reported from the border area between North and South Korea in the western Demilitarized Zone (DMZ) [5]. The number of cases steadily increased until $2000(4,142$ cases), at which point they began to gradually decrease until 2004 (864 cases). However, in 2005, 2006 and 2007, the number of reported cases increased again $(1,311$, 2,019 and 2,203 cases, respectively) [6]. Initially, the patients were South Korean soldiers or veterans that had served in the western DMZ. However, the numbers of vivax cases among civilians living in the area were also 
gradually increasing [6]. According to the WHO, vivax malaria in the Democratic People's Republic of Korea (North Korea), with 99,582 reported cases in 1999; 298,058 cases in 2001; and 34,485 cases in 2004, was more prevalent than in South Korea $[7,8]$.

Plasmodium vivax in South and North Korea has unique characteristics, such as a long incubation period (maximum 13 months), seasonal transmission (only the summer season) and it is adapted to a cold climate [3,915]: the endemic areas are covered with snow in winter season. Although the evolutionary history of $P$. vivax from other countries has recently been addressed, thus far the history of P. vivax in the Korean peninsula (South and North Korea) has not been clearly understood [1618]. Several epidemiological data showed that the reemergence of vivax malaria in South Korea would be the introduction from North Korea through the DMZ [3-8]. However, the geographical origin of $P$. vivax population in the Korean peninsula has not been determined so far. In the present study, in order to estimate geographical origin of the $P$. vivax population in the Korean peninsula, phylogeographic analysis of the $P$. vivax population in South Korea and the other populations worldwide (including a North Korean isolate) was conducted.

\section{Materials}

\section{Sample collection}

Ten blood samples were collected from vivax malaria patients who were South Korean soldiers that served in the DMZ in 1999. One blood sample was collected from a Korean visitor to Japan in 2002 . He was a veteran in the Korean army who had served in the DMZ before he visited Japan and had never been abroad before his visit $[19,20]$. The patient blood samples were preserved at $30^{\circ} \mathrm{C}$ until use. These patients were also diagnosed by microscopic examination of peripheral blood smears. This study was performed according to the ethical guidelines for epidemiological studies provided by the Ministry of Education, Culture, Sports, Science and Technology and the Ministry of Health, Labour and Welfare of Japan.

\section{DNA extraction, Polymerase chain reaction (PCR) and DNA sequencing}

The parasite DNA was extracted from the frozen whole blood samples by phenol-chloroform extraction after proteinase $\mathrm{K}$ digestion [21]. The whole mitochondrial (mt) DNA sequences (approx. $6 \mathrm{~Kb}$ ) of the $P$. vivax isolates form South Korea were amplified by PCR using three pairs of primer sets:

Pv-mt1 F (5'-TTCCACTACCAAAATATAATCTCCT3')

Pv-mt1 R (5'-CACACAAAATCACCGTTCTTATAA A-3')

Pv-mt2 F (5'-TAAATGTGCTTTAATATTATTATAG3')
Pv-mt2 R (5'-CATAATTCCATAAGAAATTAATATT3')

Pv-mt3 F (5'-ATCAACAATGACTTTATTTGGTTTA3')

Pv-mt3 R (5'-ACTATAAAACATGTGATCTAATTAC$3 '$ ), which were designed form the mt sequence of the $P$. vivax af20012 isolate [GenBank: AY791517]. Three amplified DNA fragments (approx. $2 \mathrm{~Kb}$ ) overlapped with each other. Sequencing of the PCR products was performed using an Applied Biosystems 310 Genetic Analyzer (Applied Biosystems Inc, Foster City, CA, USA), using ABI PRISM Big Dye Terminator v.3.1 (Applied Biosystems Inc, Foster City, CA, USA).

\section{Phylogenetic analysis}

MtDNA sequences (approx. $6 \mathrm{~Kb}$ ) of the 11 P. vivax isolates from South Korea (present study) [DDBJ: $\underline{\mathrm{AB} 550270}$ - $\underline{\mathrm{AB} 550280]}$ and another $282 P$. vivax isolates collected worldwide that had been deposited in the GenBank database, were used for phylogenetic analysis $[16,17]$. Mu et al [16] deposited 176 sequences [GenBank: AY791517.1-AY791692.1]. Jongwutiwes et al [17] deposited 106 sequences [GenBank: AY598035.1-AY598140.1]. DNA alignment of the whole mtDNA sequences of the $P$. vivax isolates was performed by the DNA Alignment version 1.3.0.1 computer software (Fluxus Technology Ltd.) [22]. A haplotype network was constructed based on polymorphic sites of the whole mtDNA sequences of the isolates using the Median-Joining method in the NETWORK version 4.5.1.6 computer software (Fluxus Technology Ltd.) [23].

\section{Results and Discussion}

A haplotype network was inferred by the $282 \mathrm{mtDNA}$ sequences of $P$. vivax populations that had been collected worldwide, together with the 11 South Korean isolates (Figure 1). The network tree indicated that two groups of P. vivax populations coexist in South Korea (i.e. SK group $\mathrm{A}$ and $\mathrm{B})$. The isolates of the SK group A (shown in green) were genetically identical or close to some isolates from southern China (shown in red), and those of the SK group $\mathrm{B}$ (shown in green) were also genetically identical or close to other isolates from southern China (shown in red). A neighbour-joining (NJ) tree (Additional File 1a, b) using the same data set for the network analysis were clearly demonstrated that the isolates of the SK group A were clustered with some isolates from southern China, and those of the SK group B were also clustered with other isolates from southern China. Therefore, the two clusters as defined in the NJ tree were named group A complex and group B complex, respectively. The boundaries of the two group complexes were shown in red boxes.

In a previous report, Kho et al [24] also noted the observation of two types of genotypes (i.e. SK type A and B) in some antigenic molecules of $P$. vivax in South 


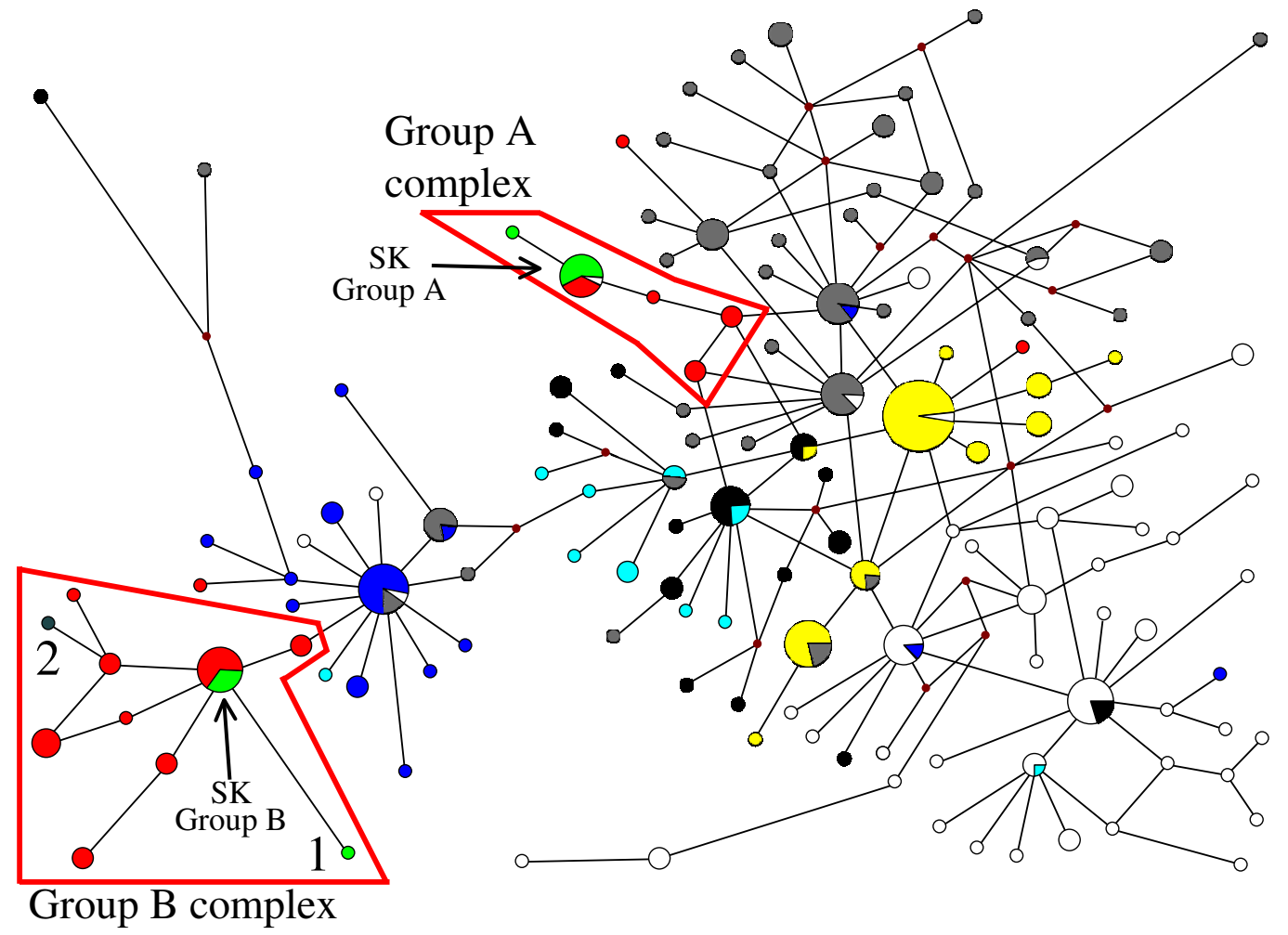

Figure 1 A haplotype network using mitochondrial genome of $P$. vivax populations. A haplotype network inferred by a median-joining method, using the 293 mitochondrial (mt) DNA sequences of P. vivax populations of which 11 are South Korean isolates (present study) and the other 282 are worldwide isolates that were deposited the GenBank database [16-18]. The size of the each circle represents the frequencies of the haplotype, with each color showing the geographical origin of the isolates. Green indicates isolates from South Korea (present study) and dark green indicates North Korea (the GenBank database). Red indicates isolates from southern China. Blue indicates isolates from Indonesia. Gray indicates isolates from Thailand, Vietnam and Bangladesh. White indicates isolates from Papua New Guinea, Vanuatu and the Solomon Islands. Black indicates isolates from India, Pakistan and Iran. Yellow indicates isolates from Central and South America. Light blue indicates isolates from Africa. No. 1 and 2 in the group B complex indicate isolates from South Korea (the first imported case into Japan from South Korea) and an isolate from North Korea, respectively.

Korean populations. In the present study, $91 \%$ of the isolates $(10 / 11)$ were correlated to the results of the previous studies on the antigenic molecules: the South Korean isolates in the group A complex had SK type A antigenic molecules, and the South Korean isolates in the group B complex had SK type B antigenic molecules (Additional File 2).

The group A complex was genetically close to Southeast Asian populations (shown in gray); most of them were isolates from Thailand and Vietnam, or close to the South Asian population (shown in black); they were isolates from India, Pakistan and Iran (Figure 1). The group B complex was genetically close to the Indonesia population (shown in blue). Generally, old (or ancestral) populations are more genetically diverged than young populations. In this context, the Southeast Asian populations (shown in gray) and Papua New Guinean populations (shown in white) seemed old populations. Considering a possible population expansion, the group $\mathrm{B}$ complex seemed to have diverged from the Indonesian population (Figure 1). The genetic divergence of the extant $P$. vivax populations in the world is presumably a result of ancient hominid geographic expansion [17]. Therefore, the relationships between the group B complex and the Indonesian population suggests a possibility that the expansion of $P$. vivax population(s) from Indonesia to southern China was brought about with the migration(s) of ancient hominids. Another possible scenario is that the group B complex has directly diverged from the Southeast Asian populations because some isolates from Southeast Asia are identical to those from Indonesia. In this scenario, the expansion of $P$. vivax population(s) from Southeast Asia to Indonesia was also brought about with the migration(s) of ancient hominids. Although the present South Korean $P$. vivax populations are believed to have recently derived from North Korea via the DMZ, this study suggests that the $P$. vivax lineages in the Korean 
peninsula have their genealogical ancestor in $P$. vivax populations from southern China.

One of the remarkable characteristics of $P$. vivax in Korean peninsula is its evolutionary adaptation to the cold climate. The long incubation period of Korean $P$. vivax is the key to the adaptation, because the parasites in the liver cells of the human host appears in the blood streams from the liver cells mainly between June and September (around the summer season) when mosquitoes are highly prevalent, but the parasites remain in the liver cells in the other colder seasons when mosquitoes are not present $[3,9,15]$. In this phenomenon, it seems as if the parasites are waiting for the mosquitoes within the host liver cells by regulating the duration of the incubation period.

Several workers reported that there seem to be two types of P. vivax strains (or populations) in the Korean peninsula: one with a short incubation period and the other with a long incubation period. The incubation period of the former type of North Korean strain is 14 days to 1 month, and the incubation period of the latter type of strain is 8 months to 13 months, as determined by experimental infection to humans through bites of the infected mosquitoes [25]. The proportion of strains (or populations) showing the short incubation period was $26.0 \%$, whereas the proportion of strains (or populations) showing the long incubation period was $74.0 \%$ [25]. One mtDNA sequence of a North Korean isolate deposited in the GenBank database was included in the present study. The North Korean isolate was shown as No. 2 (shown in dark green) in the group B complex in the haplotype network in Figure 1. The information of the incubation period of the North Korean isolate was not obtained.

One isolate from the imported patient in Japan $[19,20]$ with a long incubation period (at least eight months) was also grouped in the group B complex shown as No. 1 in the haplotype network (Figure 1). The information on the duration of the incubation period of the other 10 South Korean isolates used in this study has not been obtained thus far, but the branching patterns in the network tree appear to be related to the phenotypic characteristics of the parasites within the host.

Further study is needed to demonstrate whether the two groups of South Korean isolates (groups A and B) correlate with some clinical or epidemiological differences in the endemic areas. Haplotype network analysis using the mtDNA sequences of $P$. vivax is a useful tool for estimating the geographical origin of isolates as well as for the prediction of probable phenotypes.

\section{Conclusion}

The direct introduction of the present $P$. vivax populations to South Korea is thought to be from North Korea via the DMZ, but the true origin of the P. vivax popula- tions in the Korean peninsula is now suggested to be from the two different $P$. vivax populations in southern China.

\section{Additional material}

\begin{abstract}
Additional file 1 A neighbour-joining (NJ) tree inferred by mitochondrial DNA sequences of $P$. vivax. The NJ tree was constructed by MEGA version 3.1 (Kumar S, Tamura K, Nei M: MEGA3: Integrated Software for Molecular Evolutionary Analysis and Sequence Alignment. Bioinformatics 2004, 5:150-163) using Kimura's 2-parameter model for calculating genetic distance. Additional File 1a continued to Additional File 1b. The isolate (AY598125 Vietnam) with Asterisk $\left(^{*}\right)$ at the bottom of the Additional File 1a is identical to the isolate with Asterisk at the top of the Additional File $1 \mathrm{~b}$. The eleven South Korean isolates (present study) were clustered into either Group A complex or Group B complex in the Additional File 1b.

Additional file $\mathbf{2}$ Genotype of antigenic molecules and mitochondria of the eleven South Korean P. vivax isolates. PVCSP: P. vivax circumsporozoite protein, PVDBP: P. vivax Duffy binding protein, PVMSP-1: P. vivax merozoite surface protein-1, Conditions of PCR and DNA sequencing for antigenic molecules have been previously reported elsewhere [24]. *Genotype of the antigenic molecules of the isolate South Korea B1 was not consistent with that of the mitochondorial genome.
\end{abstract}

Competing interests

The authors declare that they have no competing interests.

\section{Authors' contributions}

MI and SYH carried out the molecular genetic studies, performed the phylogenetic analysis and drafted the manuscript. MF and SHK helped the molecular genetic studies and helped with the writing of the manuscript. TH and KT helped the phylogenetic analysis and helped with the writing of the manuscript. WGK collected the patients' blood samples, participated in the design of the study, acquisition of funding, coordination and writing of the manuscript. SK participated in the design of the study, acquisition of funding, coordination and writing of the manuscript. All authors read and approved the final manuscript.

\section{Acknowledgements}

The authors wish to thank Dr. Pannapa Susomboon, Research Institute, International Medical Center of Japan, for her technical assistance in this study. This work was supported by a Grant-in-Aid for Scientific Research (B) (19406013) from the Ministry of Education, Culture, Sports, Science, and Technology of Japan.

\section{Author Details}

1Department of Tropical Medicine and Malaria, Research Institute, National Center for Global Health and Medicine, 1-21-1 Toyama, Shinjuku, Tokyo 1628665, Japan, 2Department of Parasitology, Inje University, College of Medicine 633-165 Gaegum-dong, Busanjin-gu, Busan 614-735, Korea, ${ }^{3}$ Graduate School of Comprehensive Human Sciences, University of Tsukuba, 1-1-1 Tennodai, Tsukuba, Ibaraki 305-8577, Japan, 4Laboratory of Malariology, Research Institute for Microbial Diseases, Osaka University, Suita, Osaka 565-0871, Japan and 5 Department of Malariology, Paik Institute of Clinical Research, Inje University, College of Medicine, 633-165 Gaegum-dong, Busanjin-gu, Busan 614-735, Korea

Received: 30 March 2010 Accepted: 25 June 2010

Published: 25 June 2010

\section{References}

1. World Health Organization (WHO): World Malaria Report. Geneva: WHO; 2008.

2. Chai JY: Re-emerging Plasmodium vivax malaria in the Republic of Korea. Korean J Parasitol 1999, 37:129-143.

3. Ree HI: Unstable vivax malaria in Korea. Korean J Parasitol 2000, 38:119-138

4. Shin EH, Guk SM, Kim HJ, Lee SH, Chai JY: Trends in parasitic diseases in the Republic of Korea. Trends Parasitol 2008, 24:143-150. 
5. Chai IH, Lim Gl, Yoon SN, Oh WI, Kim SJ, Chai JY: Occurrence of tertian malaria in a male patient who has never been abroad. Korean $\mathrm{J}$ Parasitol 1994, 32:195-200. (in Korean, English abstract available)

6. Park JW, Jun G, Yeom JS: Plasmodium vivax malaria: status in the Republic of Korea following reemergence. Korean J Parasitol 2009, 47(Suppl):S39-50.

7. Han ET, Lee DH, Park KD, Seok WS, Kim YS, Tsuboi T, Shin EH, Chai JY: Reemerging vivax malaria: changing patterns of annual incidence and control programs in the Republic of Korea. Korean J Parasitol 2006, 44:285-294.

8. Choi YK, Choi KM, Park MH, Lee EG, Kim YJ, Lee BC, Cho SH, Rhie HG, Lee HS, Yu JR, Lee JS, Kim TS, Kim JY: Rapid dissemination of newly introduced Plasmodium vivax genotypes in South Korea. Am J Trop Med Hyg 2010, 82:426-432.

9. Hasegawa Y: Malaria in Korea. J Chosun Med Soc 1913, 4:53-69. (in Japanese. No English abstract available)

10. Eddleman EE, Hale WH, Snowden WM: Vivax malaria with long incubation period, Report of seven cases. US Armed Forces Med J 1951 2:1693-1698.

11. Hall WH, Loomis GW: Vivax malaria in veterans of the Korean war. A preliminary report of 25 cases. New Engl J Med 1952, 246:90-93.

12. Brunetti R: Outbreak malaria with prolonged incubation period in California, a nonendemic area. Science 1954, 119:74-75.

13. Brunetti R, Fritz RF, Hollister AC: An outbreak of malaria in California, 1952-1953. Am J Trop Med Hyg 1954, 3:779-788.

14. Tiburskaja NA, Sergiev PG, Vrublevskaja OS: Dates of onset of relapses and the duration of infection in induced tertian malaria with short and long incubation periods. Bull World Health Organ 1968, 38:447-457.

15. NMES: Malaria pre-eradication programme in Korea. Progress report, 1961-1965 Ministry of Health and Social Affairs of Korea 1966:75. (in Korean)

16. Mu J, Joy DA, Duan J, Huang Y, Carlton J, Walker J, Barnwell J, Beerli P, Charleston MA, Pybus OG, Su XZ: Host switch leads to emergence of Plasmodium vivax malaria in humans. Mol Biol Evol 2005, 22:1686-1693.

17. Jongwutiwes S, Putaporntip C, Iwasaki T, Ferreira MU, Kanbara H, Hughes AL: Mitochondrial genome sequences support ancient population expansion in Plasmodium vivax. Mol Biol Evol 2005, 22:1733-1739.

18. Cornejo OE, Escalante AA: The origin and age of Plasmodium vivax. Trends Parasitol 2006, 22:558-63.

19. Itoda I, Kaneko Y, Yasunami T, Kikuchi K, Yamaura H, Totsuka K: A case of imported Plasmodium vivax malaria from the demilitarized zone in South Korea. Kansenshogaku Zasshi 2003, 77:42-44. (in Japanese. No English abstract available)

20. Iwagami M, Itoda I, Hwang SY, Kho WG, Kano S: Plasmodium vivax PCR genotyping of the first malaria case imported from South Korea into Japan. J Infect Chemother 2009, 15:27-33.

21. Sambrook J, Russell DW: Molecular Cloning: A Laboratory Manual. 3rd edition. New York: Cold Harbor Laboratory Press; 2001.

22. DNA Alignment version 1.3.0.1 2008 [http://www.fluxusengineering.com/align.htm]. Fluxus Technology Ltd

23. Network version 4.5.1.6 2009 [http://www.fluxus-engineering.com/ netwinfo.htm]. Fluxus Technology Ltd

24. Hwang SY, Kim SH, Kho WG: Genetic characteristics of polymorphic antigenic markers among Korean isolates of Plasmodium vivax. Korean J Parasitol 2009, 47(Suppl):S51-58.

25. Tiburskaja NA, Vrublevskaja OS: The course of infection caused by the North Korean strain of Plasmodium vivax. WHO/MAL/77 1977, 895:1-19.

Submit your next manuscript to BioMed Centra and take full advantage of:

- Convenient online submission

- Thorough peer review

- No space constraints or color figure charges

- Immediate publication on acceptance

- Inclusion in PubMed, CAS, Scopus and Google Scholar

- Research which is freely available for redistribution

Submit your manuscript at www.biomedcentral.com/submit
C BioMed Central 